\title{
Baicalein inhibits inflammatory response and promotes osteogenic activity in periodontal ligament cells challenged with lipopolysaccharides
}

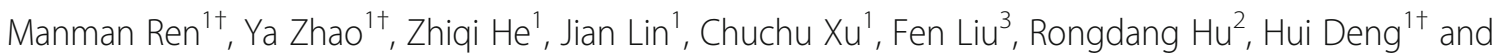
Yi Wang ${ }^{2^{*}+}$ (D)

\begin{abstract}
Background: Periodontitis is a chronic infection initiated by oral bacterial and their virulence factors, yet the severity of periodontitis is largely determined by the dysregulated host immuno-inflammatory response. Baicalein is a flavonoid extracted from Scutellaria baicalensis with promising anti-inflammatory properties. This study aims to clarify the anti-inflammatory and osteogenic effects of baicalein in periodontal ligament cells (PDLCS) treated with lipopolysaccharides (LPS).

Methods: Human PDLCs were incubated with baicalein $(0-100 \mu \mathrm{M})$ for $2 \mathrm{~h}$ prior to LPS challenge for $24 \mathrm{~h}$. MTT analysis was adopted to assess the cytoxicity of baicalein. The mRNA and protein expression of inflammatory and osteogenic markers were measured by real-time polymerase chain reaction (PCR), western blot and enzyme-linked immunosorbent assay (ELISA) as appropriate. Alkaline phosphatase (ALP) and Alizarin red S (ARS) staining were performed to evaluate the osteogenic differentiation of PDLCs. The expression of Wnt/ $\beta$-catenin and mitogenactivated protein kinase (MAPK) signaling related proteins was assessed by western blot.

Results: MTT results showed that baicalein up to $100 \mu \mathrm{M}$ had no cytotoxicity on PDLCs. Baicalein significantly attenuated the inflammatory factors induced by LPS, including interleukin-1 $\beta$ (IL-1 $\beta$ ), tumor necrosis factor-a (TNFa), matrix metalloprotein-1 (MMP-1), MMP-2 and monocyte chemoattractant protein 1 (MCP-1) at both mRNA and protein level. Moreover, MAPK signaling (ERK, JNK and p38) was significantly inhibited by baicalein, which may account for the mitigated inflammatory response. Next, we found that baicalein effectively restored the osteogenic differentiation of LPS-treated PDLCS, as shown by the increased ALP and ARS staining. Accordingly, the protein and gene expression of osteogenic markers, namely runt-related transcription factor 2 (RUNX2), collagen-I, and osterix were markedly upregulated. Importantly, baicalein could function as the $\mathrm{Wnt} / \beta$-catenin signaling activator, which may lead to the increased osteoblastic differentiation of PDLCs.
\end{abstract}

(Continued on next page)

\footnotetext{
* Correspondence: wangyi.ortho@gmail.com

${ }^{\dagger}$ Manman Ren \& Ya Zhao, Hui Deng \& Yi Wang contributed equally to this work.

${ }^{2}$ Department of Orthodontics, School of Stomatology, Wenzhou Medical University, Wenzhou, Zhejiang, China

Full list of author information is available at the end of the article
} 
(Continued from previous page)

Conclusions: With the limitation of the study, we provide in vitro evidence that baicalein ameliorates inflammatory response and restores osteogenesis in PDLCs challenged with LPS, indicating its potential use as the host response modulator for the management of periodontitis.

Keywords: Periodontitis, Baicalein, Osteogenesis, Inflammation, MAPKs, Wnt/ß-catenin

\section{Background}

Periodontitis, which affects about 5 to $15 \%$ of world populations, remain as a major global health burden. It is characterized by the accumulation of dental biofilm and resultant destruction of tooth-supporting tissues that eventually leads to multiple tooth loss $[1,2]$. Moreover, periodontitis is known to be responsible for an increased risk of a series of systemic comorbidities, such as cardiovascular disease and diabetes mellitus [3, 4].

Periodontal pathogens, which mainly comprised of Gram-negative anaerobic bacterial, are required to initiate periodontitis. However, it is the dysregulated host immuno-inflammatory response that largely determines the extent and severity of periodontal disease [5]. The infection with the pathogenic microflora, together with their virulence factors like lipopolysaccharides (LPS) and gingipain, drives the influx of neutrophils and macrophages, and activates the constituent cells, e.g. periodontal ligament cells (PDLCs), releasing a plethora of inflammatory mediators that participates in the initiation and progression of periodontitis $[5,6]$. Several signaling pathways have been implicated in the complex underlying molecular network of periodontitis. For example, mitogen-activated protein kinase (MAPK) and nuclear factor-kappaB (NF-kB) are known to regulate the expression of inflammatory mediators [7], and Wnt/ $\beta$-catenin signaling is closely involved in bone turnover and remodeling [8].

Host modulation therapy with anti-inflammatory agents has now been used as a promising adjunct to conventional periodontal management which mainly deals with the bacterial challenge [9]. Baicalin, and its aglycone baicalein are the two major bioactive flavonoids extracted from Scutellaria baicalensis Georgi (Named Huang-qin in Chinese), which are widely used in traditional Chinese herbal medicine. It is well documented that baicalein and baicalin both have diverse functional activities such as osteogenesis, cardioprotective, anticancer and anti-diabetes, mainly due to their remarkable anti-inflammation and anti-oxidant efficacies [10-13].

In periodontal research, baicalin has been shown to significantly mitigate the ligature-induced alveolar bone breakdown in rats with experimental periodontitis [14], and this could be explained by its pro-osteogenic effect on PDLCs [15]. Yet, the underlying mechanism and relevant signaling pathways are poorly characterized. On the other hand, few reports have been focused on the modulatory effects of baicalein in periodontitis. A recent study suggested that baicalein induces the proliferation and formation of calcified nodules in PDLCs, while baicalin not [16]. Moreover, nano-encapsulated baicalein, rather than baicalin, exerts pronounced anti-inflammatory effect in gingival epithelial cells under inflammatory environment [17]. The diverse effects observed between baicalein and baicalin could be explained by their different structures and bioavailability. In addition, we have previously shown that baicalein enables the osteoblastic differentiation of PDLCs through Wnt/ $\beta$-catenin signaling [18]. Therefore, this extended study aims to evaluate the effects of baicalein on the inflammatory and osteogenic factors in PDLCs challenged with LPS, as well as the underlying molecular mechanisms.

\section{Methods}

\section{Cell culture and treatment}

Human PDLCs were isolated and cultured according to our previously established protocol [19]. Cells were characterized by immunohistochemical analysis for vimentin and cytokeratin (1:200, BosterBio, China). Cells at Passage $3-4$ were used in the following experiments. This study was approved by the Ethics Committee of School \& Hospital of Stomatology, Wenzhou Medical University. Written Informed consents were obtained from all participants prior to the enrollment.

Baicalein (purity > 95\%, Sigma, USA) was dissolved in dimethylsulfoxide (DMSO, Sigma, USA), and added to the culture medium to reach the indicated concentration $(10-100 \mu \mathrm{M})$. DMSO without baicalein was used as control. $2 \mathrm{~mL}$ of PDLCs were firstly plated into 6-well plates at a density of $2 \times 10^{5}$ cells/well. After confluence, cells were incubated with baicalein for $2 \mathrm{~h}$ prior to LPS (200 ng/ml, Escherichia coli O55:B5, L2880, Sigma, USA) stimulation for $24 \mathrm{~h}$, were harvested for following analytical experiments, except that the phosphorylation of MAPK family proteins were assessed after $1 \mathrm{~h}$ of LPS treatment. The concentration of LPS was applied at 200 $\mathrm{ng} / \mathrm{ml}$ as it showed pronounced pro-inflammatory effects within the least concentration based on our preliminary results. To inhibit or activate the specific signaling pathway, cells were treated with MAPK inhibitors $(20 \mu \mathrm{M}$, PD98059, ERK inhibitor; SP600125, JNK inhibitor; SB203580, p38 inhibitor, Calbiochem, USA) or Wnt3a 
(100 ng/ml, Wnt/ $\beta$-catenin activator, Sigma, USA) for $24 \mathrm{~h}$ together with LPS.

\section{Cell viability assay}

The effects of baicalein on the proliferation of PDLCs was assessed by a 3-(4,5-dimethylthiazol-2-yl)-2,5-diphenyltetrazolium bromide (MTT) analysis. Cells were plated into 96 -well plates $\left(2 \times 10^{3}\right.$ cells/well $)$ overnight and incubated with increasing concentrations of baicalein $(0,10,20,40,60,80,100 \mu \mathrm{M})$ for $12,24,36,72$ and $96 \mathrm{~h}$ respectively. $20 \mu \mathrm{L}$ of $\mathrm{MTT}$ solution $(5 \mathrm{mg} / \mathrm{ml}$, Sigma, USA) was added to the wells and kept in dark $4 \mathrm{~h}$ prior to the indicated time. The formazan crystals were then dissolved in $150 \mu \mathrm{L}$ of DMSO and the absorbance was measured at $570 \mathrm{~nm}$ by a microplate reader (Infinite M200 PRO, Tecan, China).

Alkaline phosphate (ALP) and alizarin red S (ARS) staining Following the indicated treatment, cells were cultured in the osteogenic medium containing $10^{-5} \mathrm{mM}$ dexamethasone, $50 \mu \mathrm{g} / \mathrm{mL}$ ascorbic acid, and $5 \mathrm{mM} \beta-$ glycerophosphate for 7 days. ALP staining was subsequently performed with the use of the BCIP/NBT Alkaline Phosphatase Color Development kit as per the manufacturer's protocol (Beyotime, China). The ARS staining was conducted following 21 days of PDLCs culture in the osteogenic medium. Cells were fixed and stained with $1 \mathrm{mg} / \mathrm{ml}$ of Alizarin Red solution (Sigma, USA). The red mineralized nodules were observed under phase contrast microscope.

\section{Enzyme-linked immunosorbent assay (ELISA)}

After the indicated treatment, the culture supernatants were collected and stored under $-70^{\circ} \mathrm{C}$ until analysis. ELISA kits (Multisciences, China) was used to quantify the concentrations of interlukein-1 $\beta$ (IL-1 $\beta$ ), tumor necrosis factor- $\alpha$ (TNF- $\alpha$ ), matrix metalloprotein-1 (MMP1), MMP-2 and monocyte chemoattractant protein 1 (MCP-1) in the culture supernatants according to the manufacturer's protocol.

\section{RNA preparation and real-time quantitative polymerase chain reaction ( $R T-q P C R$ )}

Following the indicated treatment, total RNA was extracted by Trizol reagent (Invitrogen, USA). The concentration and purity of RNA was determined by spectrophotometer. An equal mass of RNA $(1 \mu \mathrm{g})$ was reverse-transcribed into cDNA with PrimeScript ${ }^{\mathrm{TM}}$ RT Reagent Kit (Takara, Japan) following the manufacturer's protocol. RT-qPCR was performed using LightCycler 480 SYBR Green I Master (Roche, USA) on a LightCycler 480 system (Roche, USA) under the following settings: $95^{\circ} \mathrm{C}$ at $30 \mathrm{~s}$ for activation, 40 cycles of $95^{\circ} \mathrm{C}$ for 5 $\mathrm{s}, 60^{\circ} \mathrm{C}$ at $20 \mathrm{~s}$ for annealing, and extension at $60^{\circ} \mathrm{C}$ for
1 min. The primers was listed in Table 1 . The relative mRNA expression was calculated using the $2^{-\Delta \Delta C t}$ method [20].

\section{Protein preparation and Western blot}

After the indicated treatment, cells were lysed in icecold RIPA buffer (Fdbio, China). Following centrifugation, the supernatant was collected and quantitatively determined with a BCA protein assay kit (Beyotime, China). $20 \mu \mathrm{g}$ of protein was separated by $10 \%$ SDSpolyacrylamide gel electrophoresis (PAGE) and transferred to polyvinylidene difluoride membrane. The membranes were then incubated with the following primary antibody at $4{ }^{\circ} \mathrm{C}$ overnight: Runt-related transcription factor 2 (RUNX2, 1:1000, No. 8486, CST, USA), $\beta$-catenin (1:1000, No. 8480, CST, USA), LEF1 (1: 1000, No. 2230, CST, USA), Cyclin D (1:1000, No. 2978, CST, USA), $\beta$-actin (1:1000, No. 4970, CST, USA), Collagen-I (COL-I, 1:1000, No. sc-8785, Santa Cruz, USA), Osterix (OSX, 1:2000, No. ab94744, Abcam, USA), ERK (1:1000, No. 4695, CST, USA), phospho-ERK (p-ERK, 1:1000, No. 4370, CST, USA), p38 (1:1000, No. 8690, CST, USA), phospho-p38 (p-p38, 1:1000, No. 4511, CST, USA), JNK (1:1000, No. 9252, CST, USA), phospho-JNK (p-JNK, 1:1000, No. 4668, CST, USA). Following the incubation with horseradish peroxidase (HRP)-conjugated secondary antibodies for $2 \mathrm{~h}$, the protein bands were visualized by Bio-Rad ChemiDocTM XRS + with ECL enhanced chemiluminescence kit. The intensity of the bands was quantified by ImageJ software (Version 1.52, JAVA, Win, NIH).

\section{Statistics}

All the experiments were performed in at least triplicate independently and the results were presented as mean \pm standard deviation (SD). The comparison was made by one-way analysis of variance (ANOVA) with post hoc LSD test. $P$ value $<0.05$ indicates statistical significance. All statistical analysis was performed on SPSS 19.0 (IBM, USA).

\section{Results}

\section{Characterization of PDLCs}

On day 5-7, PDLCs growing out of the tissues were observed under microscope (Fig. 1a). After passaging, cells displayed a spindle-like shape, which resembled the typical morphology of fibroblast cells (Fig. 1b). The immunohistochemical staining was positive for vimentin (Fig. 1c) and negative for cytokeratin (Fig. 1d). Collectively, these results suggest that the cells could be identified as PDLCs, which was consistent with previous findings $[21,22]$. 
Table 1 Primer Sequences

\begin{tabular}{|c|c|c|}
\hline Gene & Forward $\left(5^{\prime}-3^{\prime}\right)$ & Reverse $\left(5^{\prime}-3^{\prime}\right)$ \\
\hline RUNX2 & CCCGTGGCCTTCAAGGT & CGTTACCCGCCATGACAGTA \\
\hline OSX & ACCTACCCATCTGACTTTGCTC & СCACTATTTCCCACTGCCTTG \\
\hline COL-I & CCAGAAGAACTGGTACATCAGCAA & CGCCATACTCGAACTGGAATC \\
\hline $\mathbb{I L}-1 \beta$ & CGTTACCCGCCATGACAGTA & TGCTGTAGTGGTGGTCGGAGA \\
\hline TNF-a & CCTGGTATGAGCCCATCTATC & GGTTGGATGTTCGTCCTCCTC \\
\hline MMP-1 & TCGTGGTTCCAACTCGGTTT & GCGGCCCTCGAAGATGA \\
\hline MMP-2 & CCGTCGCCCATCATCAA & AGATATTGCACTGCCAACTCT \\
\hline MCP-1 & AGCATGACAAGGCCTGCGTC & TGGCACCCAGCACAATGAA \\
\hline$\beta$-actin & TGGCACCCAGCACAATGAA & CTAAGTCATAGTCCGCCTAGAAGCA \\
\hline
\end{tabular}

\section{Baicalein did not affect cell viability in PDLCs}

As shown in Fig. 2, MTT assay showed that baicalein at concentrations ranging from 10 to $100 \mu \mathrm{M}$ did not affect cell viability up to $96 \mathrm{~h}$, indicating a good biosafety.

\section{Baicalein suppressed inflammation and MAPK signaling in LPS-treated PDLCs}

As indicated in Fig. 3a-d, 20, 40 and $80 \mu \mathrm{M}$ of baicalein all effectively downregulated the LPS-induced transcripts and supernatant concentrations of inflammatory cytokines including IL- $1 \beta$, TNF- $\alpha$ and MCP-1 $(P<0.05)$. While the mRNA and protein expressions of MMP-1 and MMP-2 were found to be significantly decreased by the pre-treatment of baicalein at the concentrations of 40 and $80 \mu \mathrm{M}(P<0.05)$. The immunoblotting results showed that treatment with LPS for $1 \mathrm{~h}$ markedly induced the phosphorylation of the MAPK family proteins, namely ERK, JNK and p38 $(P<0.05$, Fig. 4a). Moreover, the mRNA and protein expressions of inflammatory factors (IL-1 $\beta$, MCP-1, MMP-1 and -2) up-regulated by LPS could be significantly attenuated by the selective inhibitor of ERK, JNK and p38 respectively $(P<0.05)$. The transcripts and protein level of TNF- $\alpha$ were reduced via the inhibition of ERK and JNK signaling only (Fig. 4b \& c). Importantly, baicalein impeded the phosphorylation of ERK, JNK and p38 induced by LPS (Fig. 4a). Given the effect of MAPK inhibitors on the suppression of
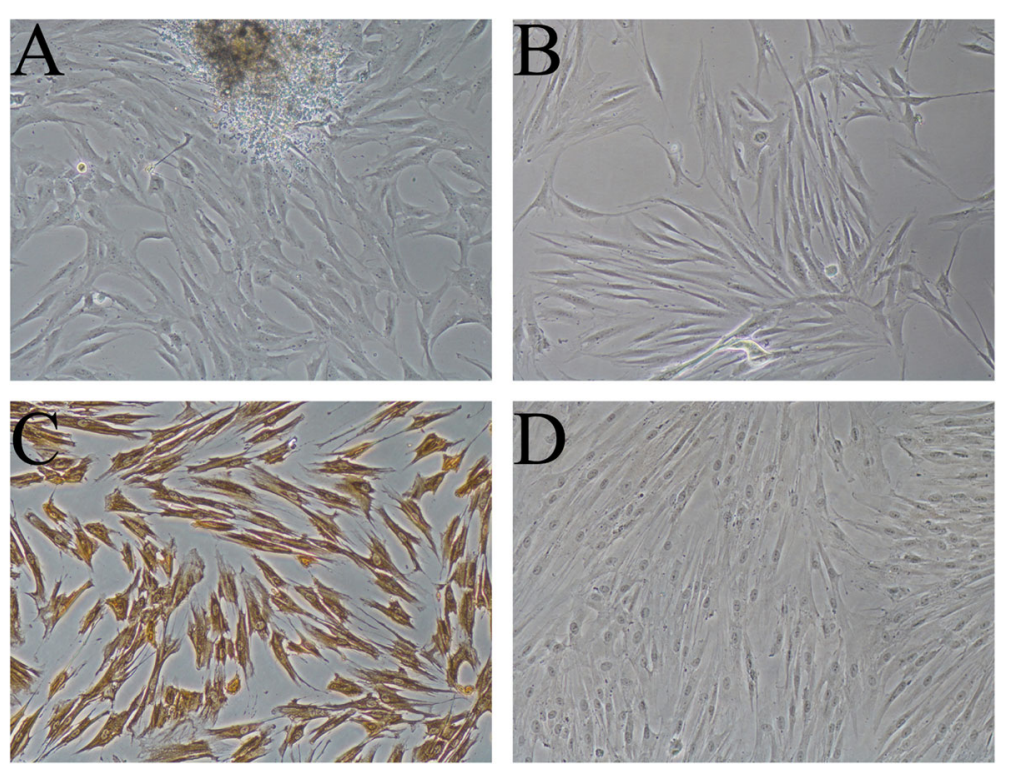

Fig. 1 The characterization of PDLCs. Primary culture of PDLCs at the Day $7(\times 100)(\mathbf{a})$; Morphology of PDLCs at Passage $1(\times 100)(\mathbf{b})$; Immunohistochemistry staining results of PDLCs, positive for vimentin $(\times 100)(\mathbf{c})$ and negative for cytokeratin $(\times 100)(\mathbf{d})$ 


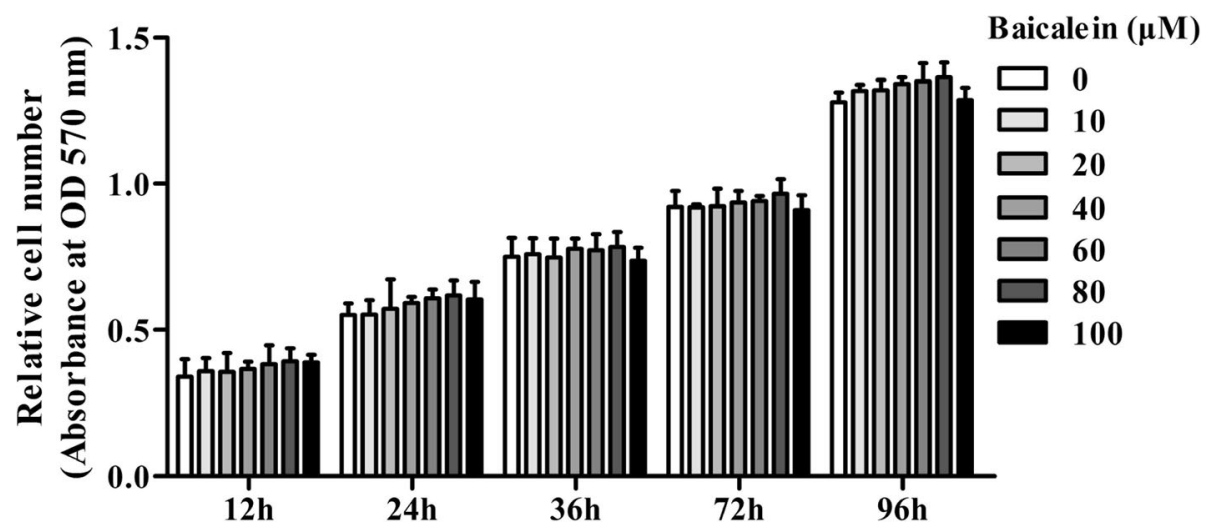

Fig. 2 The effects of baicalein on the viability of PDLCs determined by the MTT assay. It shows that baicalein (20, 40, or $80 \mu M)$ did not affect the cell viability up to $96 \mathrm{~h}$

inflammation, it is suggested that baicalein may mitigate the expressions of inflammatory factors in LPStreated PDLCs through MAPK signaling inhibition. The uncropped blots for Fig. 4a can be viewed in Fig. S1 in the online supplementary materials.

\section{Baicalein promotes osteogenesis and $\mathrm{Wnt} / \beta$-catenin signaling in LPS-treated PDLCs}

The early and late osteogenic differentiation of PDLCs was assessed by ALP and ARS assay respectively. It was shown that the baicalein markedly increased the intensity of ALP staining and the number of red mineralized nodules in LPS-treated cells (Fig. 5a \& b).
Accordingly, the mRNA and protein expressions of osteogenic markers including COL-I, RUNX2 and OSX, which were downregulated by LPS, were significantly recovered by baicalein at 40 and $80 \mu \mathrm{M}(P<$ 0.01 , Fig. $5 \mathrm{c} \& \mathrm{~d}$ ). The uncropped blots for Fig. $5 \mathrm{~d}$ were shown in Fig. S2 in the online supplementary materials.

The modulatory effect of $\mathrm{Wnt} / \beta$-catenin signaling in LPS-treated PDLCs was further investigated. We found that LPS reduced the expression of $\mathrm{Wnt} / \beta$-catenin signaling related proteins including $\beta$-catenin, LEF1 and Cyclin D (Fig. 6a). Furthermore, the activation of $\mathrm{Wnt} / \beta$-catenin signaling by Wnt3a

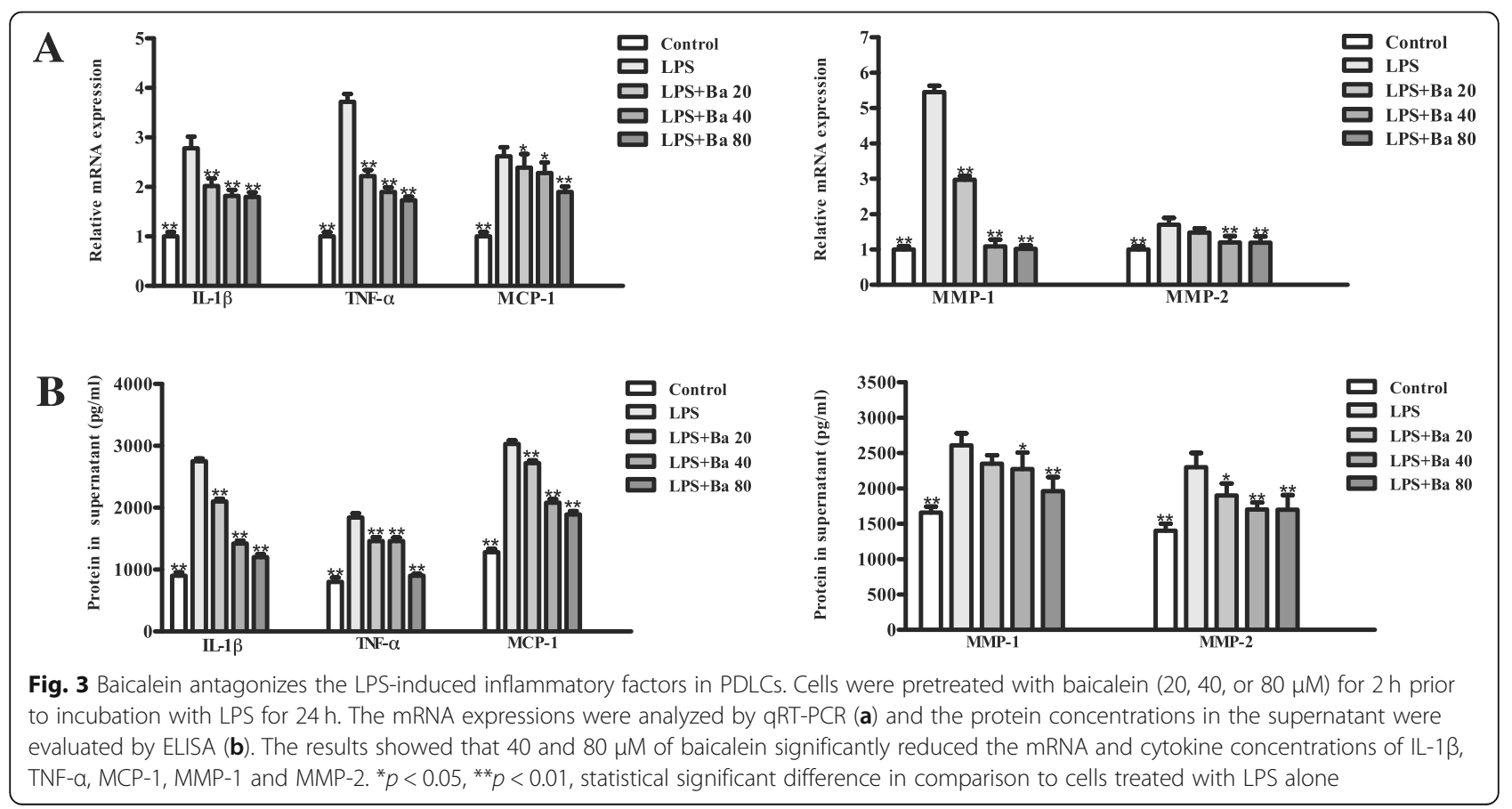




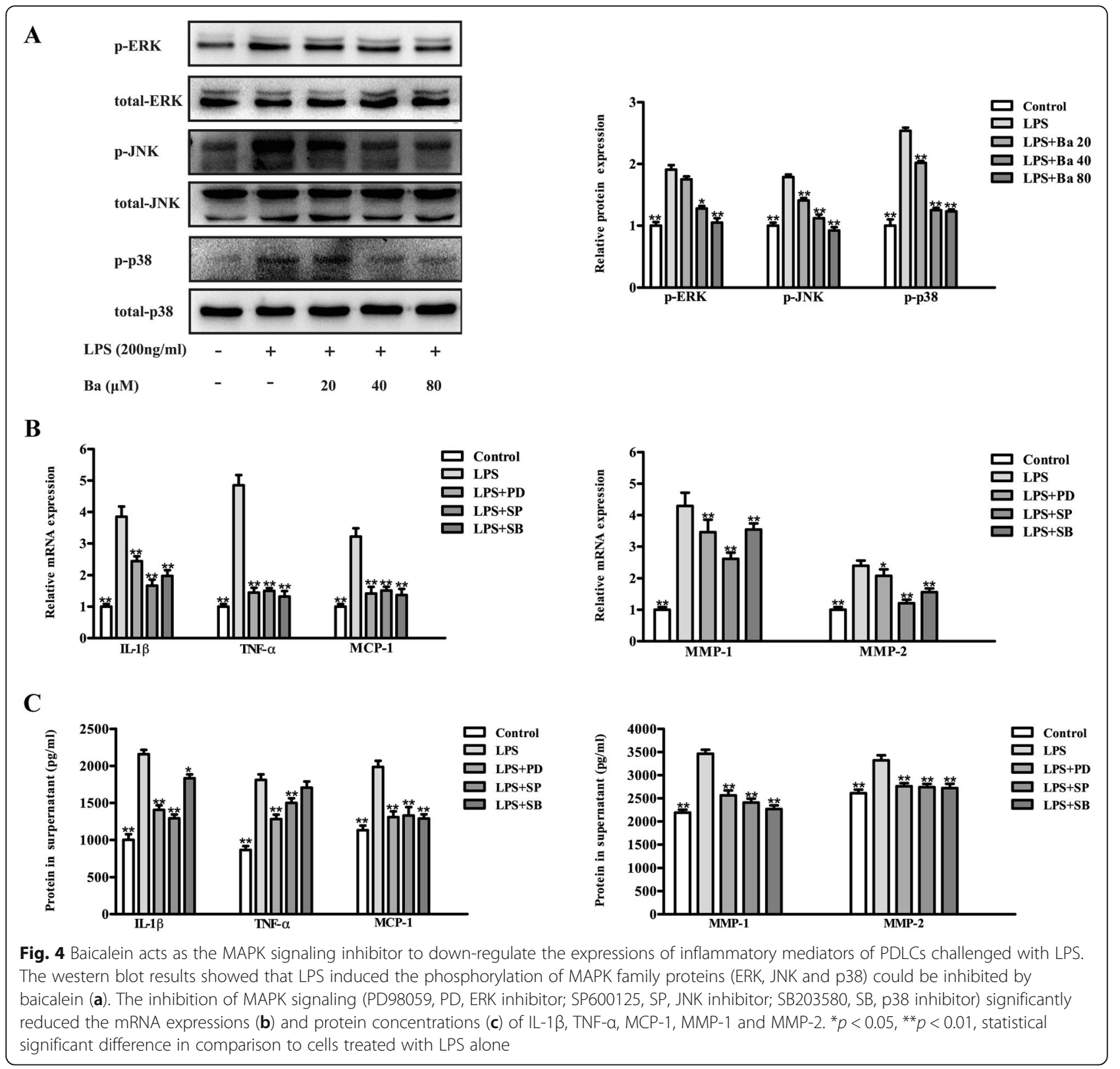

significantly increased the osteogenic differentiation of PDLCs treated with LPS (Fig. 6b \& c), accompanied by the elevated mRNA and protein expressions of osteogenic markers (COL-I, RUNX2 and OSX, $P<0.05$, Fig. $6 \mathrm{~d} \&$ e). Provided that baicalein might act as a Wnt $/ \beta$-catenin signaling activator to induce the protein expressions of $\beta$-catenin, LEF1 and Cyclin D (Fig. 6a), we assumed that baicalein could recover the osteogenic differentiation of LPS-treated PDLCs via the activation of $\mathrm{Wnt} / \beta$-catenin signaling. The uncropped blots for Fig. $6 a$ and e were presented in Fig. S3 and 4 respectively in the online supplementary materials.

\section{Discussion}

Periodontitis is essentially the consequence of the dysregulated host-microbial interactions. Recently, therapeutic management towards the host immunoinflammatory response has been proposed as a promising approach [23]. Indeed, several compounds from Chinese medicinal herbs have been suggested for the treatment of periodontal disease, e.g., osthole from Cnidium monnieri [24], shikonin from Radix Arnebiae [25], psoralen and angelicin from Psoraleae [26]. The present study demonstrated that baicalein increased osteogenic differentiation and decreased inflammation in LPS-treated PDLCs, suggesting that baicalein could 


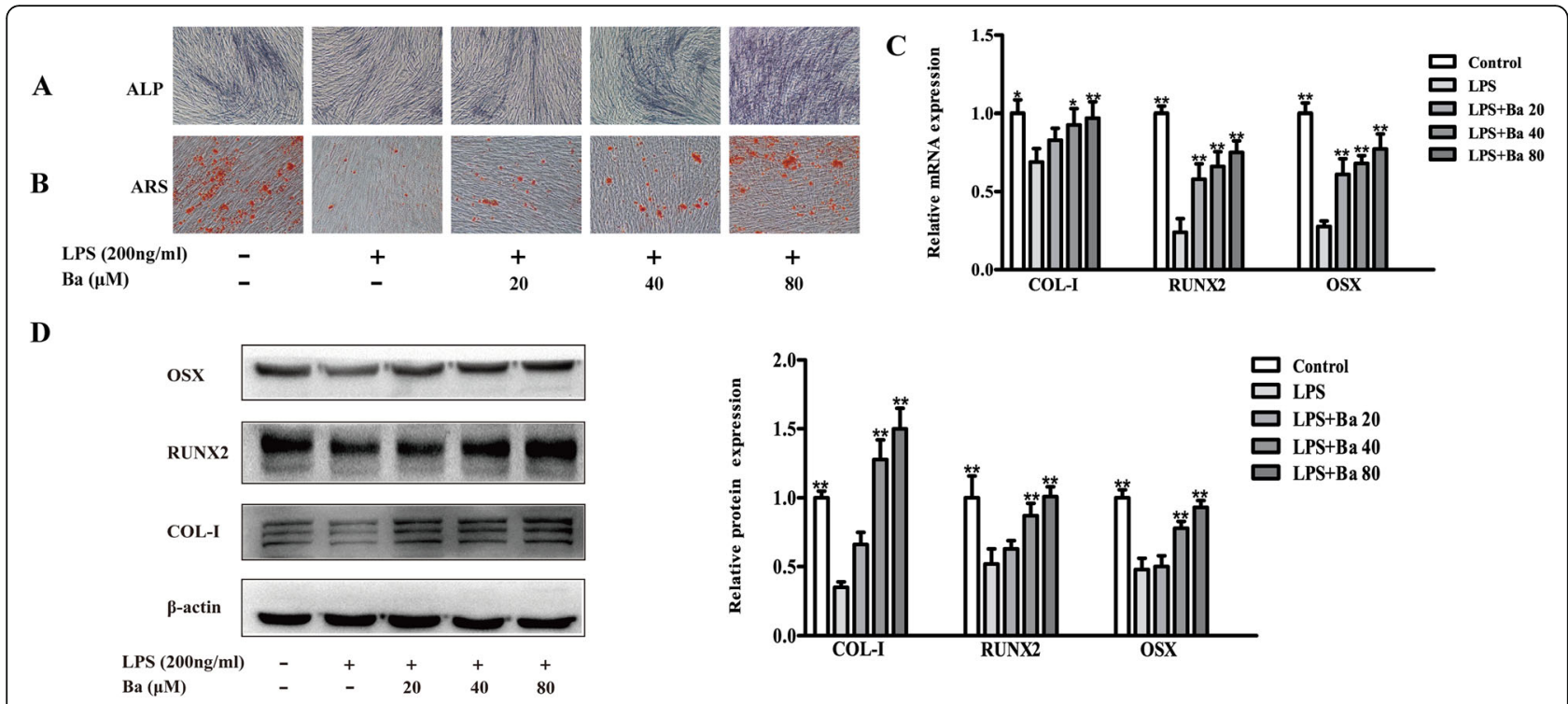

Fig. 5 Baicalein restores the osteogenesis of PDLCs challenged with LPS. Cells were pre-treated with baicalein (20, 40, or $80 \mu \mathrm{M})$ for $2 \mathrm{~h}$ prior to incubation with or without LPS for $24 \mathrm{~h}$. Baicalein effectively increased the ALP (a) and ARS (b) staining of LPS-treated PDLCs. The mRNA (c) and protein (d) expressions of COL-1, RUNX2 and OSX were dose-dependently up-regulated by baicalein. ${ }^{*} p<0.05,{ }^{* *} p<0.01$, statistical significant difference in comparison to cells treated with LPS alone.

be potentially used in addition to dental biofilm management.

PDLCs are closely involved in repair, remodeling and regeneration of the adjacent cementum and bone, contributing to the maintenance of tooth supporting tissues. It is demonstrated that PDLCs exhibit a decreased osteogenicity in the presence of inflammatory microenvironment, resulting in the breakdown of the adjacent tissues [27]. E. coli-LPS is a well-known potent inducer of inflammatory cytokines and chemokines, and therefore used to mimic microenvironment in the present study. Our study showed that LPS inhibited the osteogenic differentiation of PDLCS, together with a markedly increased secretion of inflammatory mediators, confirming the results of previous studies [28, 29].

The inflammatory cytokines play a crucial role in the host response to periodontal infection. Clinical evidence suggested that the gingival crevicular levels of IL-1 $\beta$ and TNF- $\alpha$ are positively associated with the onset and progression of periodontitis [30]. In addition, an increased level MCP-1 enables the immune cells to infiltrate and accumulate into the gingival sulcus, producing a plethora of pro-inflammatory cytokines. These cytokines may in turn amplify the chemotactic signals that generated by PDLCs challenged with inflammatory agents [5]. Moreover, MMP-1 and -2 are important enzymes for matrix degradation. Their upregulation are responsible for the turnover of periodontal ligament collagens and subsequent attachment loss [31]. The anti-inflammatory property of baicalein has been validated in immune cells, endothelial cells and oral epithelial cells [17, 32, 33], whether such effect could be extended to PDLCs remains to be clarified. To the best of our knowledge, the present study for the first time, showed that baicalein effectively reduced these inflammatory mediators (IL-1 $\beta$, TNF- $\alpha$, MCP-1, MMP-1 \& -2) induced by LPS in PDLCs.

MAPK signaling has been widely reported to be the potential target for inflammatory stimuli [34]. Indeed, pro-inflammatory agents have been demonstrated to induce the phosphorylation of MAPK family proteins including ERK1/2, JNK and p38 in PDLCs [35, 36]. Accordingly, we found that the inhibition of ERK1/2, JNK and p38 pathways resulted in an reduced expression of inflammatory cytokines. Importantly, baicalein impeded the MAPK signaling activated by LPS, potentially suggesting that the anti-inflammatory effect was mediated by MAPK signaling. Moreover, the suppression of MAPK signaling by baicalein has been demonstrated to be regulated by the upstream Toll-like Receptor-4 (TLR4) $[37,38]$. Since TLR $2 / 4$ are highly functional on PDLCs exposed to gram-negative bacterial challenge [39], future studies investigating the TLR-MAPK axis are highly warranted.

The osteogenic effects of baicalein is controversial. It is reported that baicalein slightly suppressed the ALP activity of MC3TE-1 cells [40]. While another group consistently demonstrated that baicalein promotes the osteoblastic differentiation in MC3TE-1, and inhibits the bone resorption by inducing the apoptosis of mature osteoclasts [41, 42]. Moreover, since the regeneration capacity of inflamed PDLCs is impaired, it is important to 
A

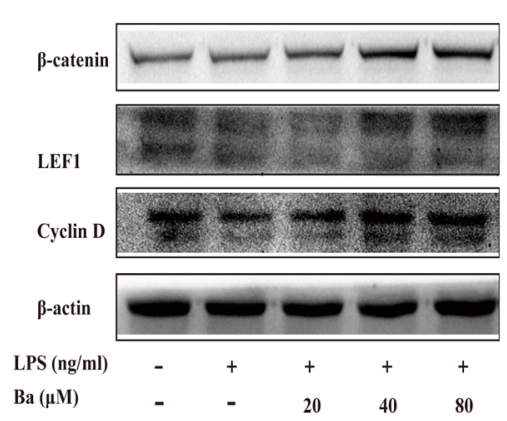

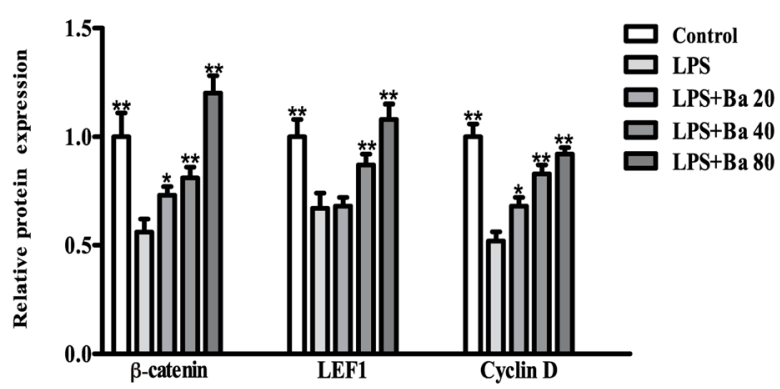
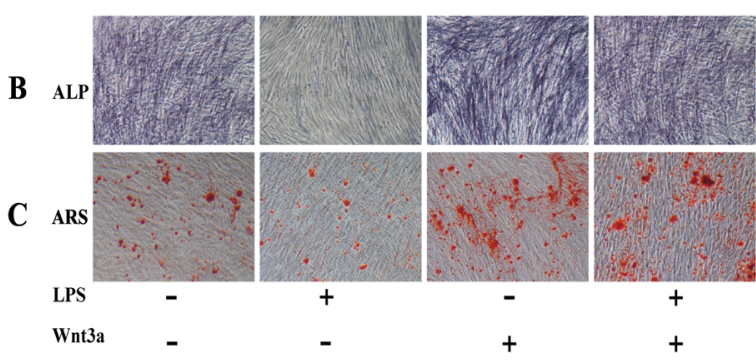

Wnt3a

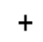

E
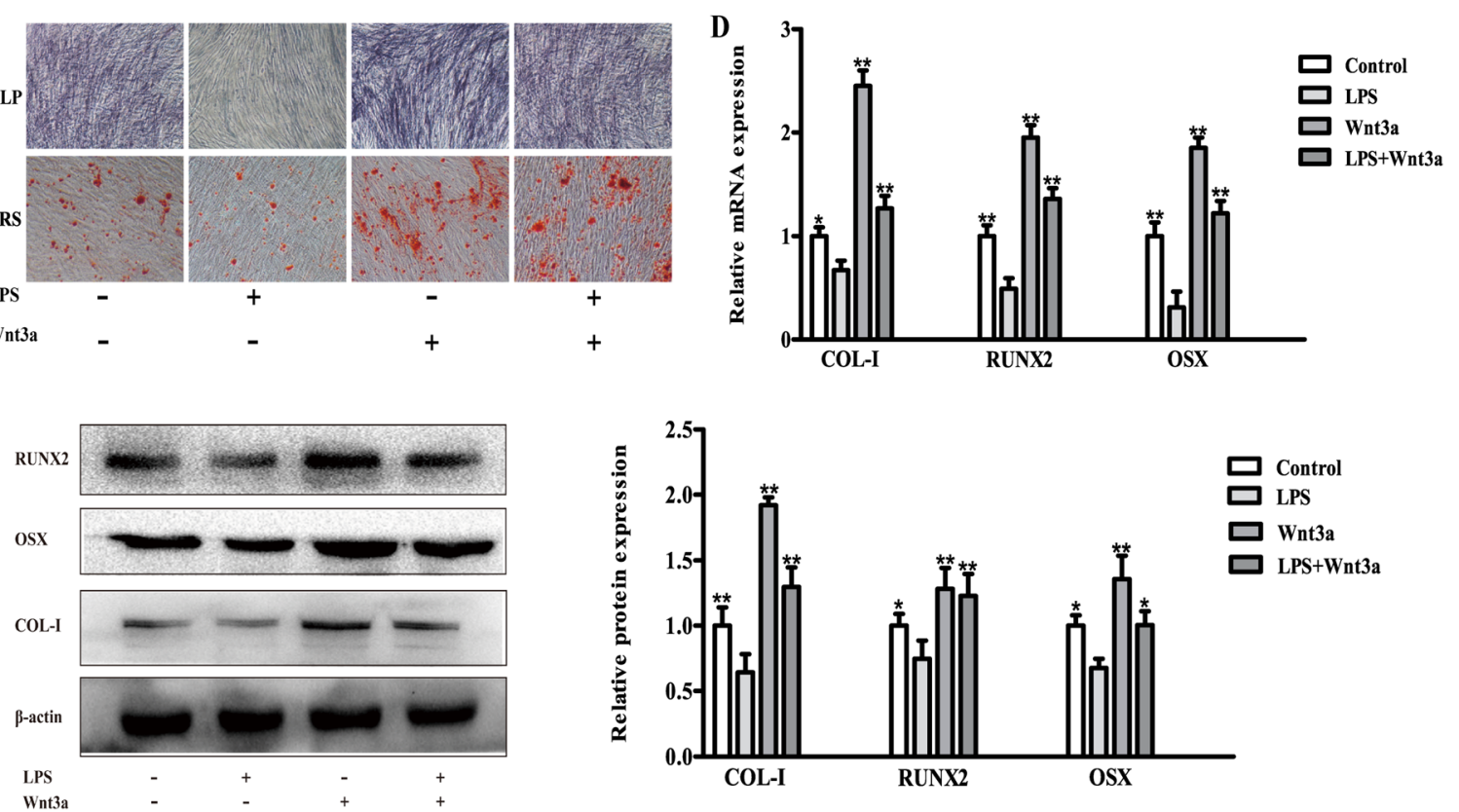

Fig. 6 Baicalein acts as the Wnt/ $\beta$-catenin signaling activator to up-regulate the osteogenesis of PDLCS challenged with LPS. The LPS-decreased expression of Wnt/ $\beta$-catenin signaling related protein was recovered by baicalein (a). The ALP staining (b) and mineralized nodule formation (c) inhibited by LPS, was found to be restored by Wnt/ $\beta$-catenin signaling activator, wnt3a. The mRNA (d) and protein (e) expressions of COL-1, RUNX2 and OSX, which were down-regulated by LPS, were significantly increased following the treatment of baicalein. ${ }^{*} p<0.05$, ${ }^{* *} p<0.01$, statistical significant difference in comparison to cells treated with LPS

investigate the osteogenic effect of baicalein under inflammatory microenvironment [43]. ALP staining (indicator of early phase osteoblastic differentiation) and mineralized nodules (indicator of late phase osteoblastic differentiation) were found to be markedly increased by baicalein in LPS-challenged cells in our study. RUNX-2, is a master regulator of osteoblastic differentiation, who serves as the upstream activator of OSX and COL-1. Osterix is actively involved in the maturity and terminal differentiation of osteoblast, and collagen-I is a bone protein secreted exclusively by osteoblasts $[44,45]$. Our result showed that baicalein dose-dependently up-regulated the transcripts and protein expressions of RUNX-2, OSX and COL-1 in LPS-challenged cells, which were in accordance with ALP and ARS findings.
It is well acknowledged that activation of $\mathrm{Wnt} / \beta$-catenin promotes osteoblastic differentiation, while the disruption of which leads to impaired osteogenesis [46]. Besides, RUNX2, which was found to be elevated by baicalein, is a direct target of canonical Wnt signaling [47]. We therefore investigated the effects of baicalein on $\mathrm{Wnt} / \beta$-catenin signaling. In the present study, osteogenic capacity reduced by LPS could be almost completely reversed by the addition of Wnt3a, a potent activator of Wnt/ $\beta$-catenin signaling. Notably, Wnt targeted proteins (LEF1, Cyclin D and $\beta$-catenin) were significantly induced by baicalein, suggesting that baicalein may function as $\mathrm{Wnt} / \beta$-catenin signaling activator, promoting the osteogenicity of PDLCs under inflammatory microenvironment. This was in accordance with previous studies in which agents that activating Wnt/ $\beta$ - 
catenin signaling could stimulate PDLCs osteoblastic differentiation in the inflammatory environment $[48,49]$.

The present study indicated that $2 \mathrm{~h}$ of pre-treatment with baicalein effectively attenuated the LPS-induced inflammatory factors, as well as the LPS-inhibited osteogenic differentiation in PDLCs. Whereas it may take 24 to $48 \mathrm{~h}$ of co-incubation with baicalin to exert its protective effect on PDLCs, as indicated by previous studies $[50,51]$. Given the low half-life and rapid elimination from the local sites in the oral cavity for baicalein and baicalin [17], a short period of time $(2 \mathrm{~h})$ may better mimic the in vivo conditions. Nevertheless, future studies are highly warranted to verify the in vivo effects and relevant molecular mechanisms of baicalein in the treatment of periodontitis. Moreover, the in vivo efficacy and biosafety of baicalein, in comparison to baicalin and other chemical anti-inflammation agents, should be investigated to determine the potential candidate for clinical use.

Several limitations have to be addressed. LPS derived from $E$. coli was adopted to create inflammatory environment in our study. Although LPS originated from $E$. coli and the major periodontal pathogen $P$. gingivalis, show somewhat different molecular structure, both of them could induce the production of inflammatory cytokines in PDLCs, such as IL-6, IL-8 and MCP-1 [52, 53]. Indeed, E. coli derive LPS have been applied to oral cells in a plenty of studies to represent the inflammatory state of periodontal disease [54-56]. The osteogenesis of PDLCs in inflammatory environment is complex, involving an array of transcription factors, signaling pathways. This study only investigated a subset of pathways involved in the inflammation and osteogenesis. Future studies are needed to investigate the comprehensive signaling network in an attempt to better elucidate the underlying mechanisms of baicalein.

\section{Conclusions}

In conclusion, the present study demonstrated that baicalein antagonizes inflammation together with the inhibition of MAPK signaling, and induces osteogenicity and Wnt/ $\beta$-catenin signaling in PDLCs challenged with LPS. These results revealed the potential therapeutic implication of baicalein as the host response modulator for the treatment of periodontitis.

\section{Supplementary Information}

The online version contains supplementary material available at https://doi. org/10.1186/s12906-021-03213-5.

\section{Additional file 1}

\section{Abbreviations}

PDLCs: periodontal ligament cells,: LPS: lipopolysaccharides; PCR: polymerase chain reaction; ELISA: enzyme-linked immunosorbent assay; ALP: alkaline phosphatase; ARS: alizarin red S; IL: interleukin; TNF-a: tumor necrosis factora; MMP: matrix metalloprotein; MCP: monocyte chemoattractant protein; RUNX2: runt-related transcription factor 2; MAPK: mitogen-activated protein kinase; NF-kB: nuclear factor-kappaB; DMSO: dimethylsulfoxide;

PAGE: polyacrylamide gel electrophoresis; HRP: horseradish peroxidase; ANOVA: one-way analysis of variance; SD: standard deviation; TLR: toll-like Receptor

\section{Acknowledgements}

Not applicable.

\section{Authors' contributions}

YW, HD and RH conceived the study. MR, YZ, ZH, JL, CX and FL performed the experiments. YW, HD, RH and MR analyzed the data, YW, HD and MR wrote the manuscript. All authors read and approved the final manuscript.

\section{Funding}

This study was supported by grants from the Zhejiang Provincial Nature Science Foundation (LQ20H140002); Zhejiang Provincial Science and Technology Project for Public Welfare (LGF18H140007, LGF19H140005 \& LGF2OH140003); Zhejiang Provincial Traditional Chinese Medicine Science and Technology Plan (2017ZB068) and Public Welfare Science and

Technology Plan of Wenzhou City (Y20160140 \& Y20190100). The founders had no role in study design, data collection and analysis, decision to publish, or preparation of the manuscript.

\section{Availability of data and materials}

The datasets used and/or analyzed during the current study are available from the corresponding author on reasonable request.

\section{Ethics approval and consent to participate}

The use of human periodontal ligament cells was approved by the Ethics Committee of School \& Hospital of Stomatology, Wenzhou Medical University (Register No. 2017001). Informed consents were obtained from all participants prior to the enrollment.

\section{Consent for publication}

Not applicable.

\section{Competing interests}

The authors declare that they have no competing interests.

\section{Author details}

${ }^{1}$ Department of Periodontics, School of Stomatology, Wenzhou Medical University, Wenzhou, Zhejiang, China. ${ }^{2}$ Department of Orthodontics, School of Stomatology, Wenzhou Medical University, Wenzhou, Zhejiang, China. ${ }^{3}$ Department of Histology and Embryology, Wenzhou Medical University, Wenzhou, Zhejiang, China.

Received: 18 December 2019 Accepted: 10 January 2021 Published online: 23 January 2021

\section{References}

1. Petersen PE, Ogawa $\mathrm{H}$. The global burden of periodontal disease: towards integration with chronic disease prevention and control. Periodontol 2000 2012;60(1):15-39.

2. Pihlstrom BL, Michalowicz BS, Johnson NW. Periodontal diseases. Lancet. 2005:366(9499):1809-20.

3. Jin LJ, Lamster IB, Greenspan JS, Pitts NB, Scully C, Warnakulasuriya S. Global burden of oral diseases: emerging concepts, management and interplay with systemic health. Oral Dis. 2016;22(7):609-19.

4. Zhao D, Zhen Z, Pelekos G, Yiu KH, Jin L. Periodontal disease increases the risk for onset of systemic comorbidities in dental hospital attendees: an 18year retrospective cohort study. J Periodontol. 2019;90(3):225-33.

5. Kornman KS. Mapping the pathogenesis of periodontitis: a new look. J Periodontol. 2008;79(8 Suppl):1560-8.

6. Offenbacher S. Periodontal diseases: pathogenesis. Ann Periodontol. 1996; 1(1):821-78

7. Patil CS, Kirkwood KL. p38 MAPK signaling in oral-related diseases. J Dent Res. 2007;86(9):812-25. 
8. Yin X, Li J, Salmon B, Huang L, Lim WH, Liu B, Hunter DJ, Ransom RC, Singh $\mathrm{G}$, Gillette $\mathrm{M}$, et al. Wnt signaling and its contribution to craniofacial tissue homeostasis. J Dent Res. 2015;94(11):1487-94.

9. Preshaw PM, et al. Periodontol 2000. 2018;76(1):131-49.

10. Chang WT, Li CQ, Hsu CW, Lee C, Huang HH, Yuan CS, Chen WJ, Vanden Hoek TL, Shao ZH, Li J. Baicalein Cardioprotection via oxidant scavenging and Akt-nitric oxide signaling: identification of early reperfusion phase as the critical therapeutic window. Am J Chin Med. 2019;47(5):1043-56.

11. Dinda B, Dinda S, DasSharma S, Banik R, Chakraborty A, Dinda M. Therapeutic potentials of baicalin and its aglycone, baicalein against inflammatory disorders. Eur J Med Chem. 2017;131:68-80.

12. Fu Y, Luo J, Jia Z, Zhen W, Zhou K, Gilbert E, Liu D. Baicalein protects against type 2 diabetes via promoting islet beta-cell function in obese diabetic mice. Int J Endocrinol. 2014;2014:846742.

13. Gong WY, Zhao ZX, Liu BJ, Lu LW, Dong JC. Exploring the chemopreventive properties and perspectives of baicalin and its aglycone baicalein in solid tumors. Eur J Med Chem. 2017;126:844-52.

14. Cai X, Li C, Du G, Cao Z. Protective effects of baicalin on ligature-induced periodontitis in rats. J Periodontal Res. 2008;43(1):14-21.

15. Pei Z, Wang B, Zhang F, Niu Z, Shi S, Cannon RD, Mei L. Response of human periodontal ligament cells to baicalin. J Periodontol. 2014;85(9):1283-90.

16. Liu J, Wang S, Sun J, Shi J, Li Y, Gou J, Li A, He L. Screening of osteoanagenesis-active compounds from Scutellaria baicalensis Georgi by hPDLC/CMC-online-HPLC/MS. Fitoterapia. 2014;93:105-14.

17. Li X, Luo W, Ng TW, Leung PC, Zhang C, Leung KC, Jin L. Nanoparticleencapsulated baicalein markedly modulates pro-inflammatory response in gingival epithelial cells. Nanoscale. 2017;9(35):12897-907.

18. Chen LJ, Hu BB, Shi XL, Ren MM, Yu WB, Cen SD, Hu RD, Deng H. Baicalein enhances the osteogenic differentiation of human periodontal ligament cells by activating the Wnt/beta-catenin signaling pathway. Arch Oral Biol. 2017;78:100-8.

19. Sun C, Chen L, Shi X, Cao Z, Hu B, Yu W, Ren M, Hu R, Deng H. Combined effects of proinflammatory cytokines and intermittent cyclic mechanical strain in inhibiting osteogenicity in human periodontal ligament cells. Cell Biol Int. 2016;40(9):999-1007.

20. Livak KJ, Schmittgen TD. Analysis of relative gene expression data using real-time quantitative PCR and the 2(-Delta Delta C(T)) method. Methods. 2001;25(4):402-8.

21. Pang Y, Yuan X, Guo J, Wang X, Yang M, Zhu J, Wang J. The effect of liraglutide on the proliferation, migration, and osteogenic differentiation of human periodontal ligament cells. J Periodontal Res. 2019;54(2):106-14.

22. Wang $Y$, Hu B, Hu R, Tong X, Zhang M, Xu C, He Z, Zhao Y, Deng H. TAZ contributes to osteogenic differentiation of periodontal ligament cells under tensile stress. J Periodontal Res. 2020;55(1):152-60.

23. Sulijaya B, Takahashi N, Yamazaki K. Host modulation therapy using antiinflammatory and antioxidant agents in periodontitis: a review to a clinical translation. Arch Oral Biol. 2019;105:72-80.

24. Sun J, Dong Z, Zhang Y, He X, Fei D, Jin F, Yuan L, Li B, Jin Y. Osthole improves function of periodontitis periodontal ligament stem cells via epigenetic modification in cell sheets engineering. Sci Rep. 017;7(1):5254

25. Fan C, Zhang X, Upton Z. Anti-inflammatory effects of shikonin in human periodontal ligament cells. Pharm Biol. 2018;56(1):415-21.

26. Li X, Yu C, Hu Y, Xia X, Liao Y, Zhang J, Chen H, Lu W, Zhou W, Song Z. New application of Psoralen and Angelicin on periodontitis with antibacterial, anti-inflammatory, and Osteogenesis effects. Front Cell Infect Microbiol. 2018:8:178.

27. Liu N, Shi S, Deng M, Tang L, Zhang G, Liu N, Ding B, Liu W, Liu Y, Shi H, et al. High levels of beta-catenin signaling reduce osteogenic differentiation of stem cells in inflammatory microenvironments through inhibition of the noncanonical Wnt pathway. J Bone Miner Res. 2011;26(9):2082-95.

28. Wada N, Maeda H, Yoshimine Y, Akamine A. Lipopolysaccharide stimulates expression of osteoprotegerin and receptor activator of NF-kappa B ligand in periodontal ligament fibroblasts through the induction of interleukin-1 beta and tumor necrosis factor-alpha. Bone. 2004;35(3):629-35.

29. Wei L, Jiang Y, Zhou W, Liu S, Liu Y, Rausch-Fan X, Liu Z. Strontium ion attenuates lipopolysaccharide-stimulated proinflammatory cytokine expression and lipopolysaccharide-inhibited early osteogenic differentiation of human periodontal ligament cells. J Periodontal Res. 2018:53(6):999-1008.

30. Ertugrul AS, Sahin H, Dikilitas A, Alpaslan N, Bozoglan A. Comparison of CCL28, interleukin-8, interleukin-1 beta and tumor necrosis factor-alpha in subjects with gingivitis, chronic periodontitis and generalized aggressive periodontitis. J Periodontal Res. 2013;48(1):44-51.

31. Xiang J, Li C, Dong W, Cao Z, Liu L. Expression of matrix metalloproteinase1, matrix metalloproteinase-2 and extracellular metalloproteinase inducer in human periodontal ligament cells stimulated with interleukin-1 beta. J Periodontal Res. 2009;44(6):784-93.

32. Fan GW, Zhang Y, Jiang X, Zhu Y, Wang B, Su L, Cao W, Zhang H, Gao X. Anti-inflammatory activity of baicalein in LPS-stimulated RAW264.7 macrophages via estrogen receptor and NF-kappaB-dependent pathways. Inflammation. 2013;36(6):1584-91.

33. Lee W, Ku SK, Bae JS. Anti-inflammatory effects of Baicalin, Baicalein, and Wogonin in vitro and in vivo. Inflammation. 2015;38(1):110-25.

34. Kyriakis JM, Avruch J. Mammalian mitogen-activated protein kinase signal transduction pathways activated by stress and inflammation. Physiol Rev. 2001:81(2):807-69.

35. Jiang $L$, Song J, Hu X, Zhang H, Huang E, Zhang Y, Deng F, Wu X. The proteasome inhibitor Bortezomib inhibits inflammatory response of periodontal ligament cells and ameliorates experimental periodontitis in rats. J Periodontol. 2017;88(5):473-83.

36. Luo LJ, Liu F, Lin ZK, Xie YF, Xu JL, Tong QC, Shu R. Genistein regulates the IL-1 beta induced activation of MAPKs in human periodontal ligament cells through G protein-coupled receptor 30. Arch Biochem Biophys. 2012;522(1): 9-16.

37. Chen H, Zhang Y, Zhang W, Liu H, Sun C, Zhang B, Bai B, Wu D, Xiao Z, Lum $\mathrm{H}$, et al. Inhibition of myeloid differentiation factor 2 by baicalein protects against acute lung injury. Phytomedicine. 2019;63:152997.

38. Luo X, Yu Z, Deng C, Zhang J, Ren G, Sun A, Mani S, Wang Z, Dou W. Baicalein ameliorates TNBS-induced colitis by suppressing TLR4/MyD88 signaling cascade and NLRP3 inflammasome activation in mice. Sci Rep. 2017;7(1):16374

39. Sun Y, Shu R, Li CL, Zhang MZ. Gram-negative periodontal bacteria induce the activation of toll-like receptors 2 and 4 , and cytokine production in human periodontal ligament cells. J Periodontol. 2010;81(10):1488-96.

40. Lai CH, Wu YW, Yeh SD, Lin YH, Tsai YH. Effects of 6-Hydroxyflavone on osteoblast differentiation in MC3T3-E1 cells. Evid Based Complement Alternat Med. 2014:2014:924560.

41. Kim JM, Lee SU, Kim YS, Min YK, Kim SH. Baicalein stimulates osteoblast differentiation via coordinating activation of MAP kinases and transcription factors. J Cell Biochem. 2008;104(5):1906-17.

42. Kim MH, Ryu SY, Bae MA, Choi JS, Min YK, Kim SH. Baicalein inhibits osteoclast differentiation and induces mature osteoclast apoptosis. Food Chem Toxicol. 2008;46(11):3375-82.

43. Xia Y, Tang HN, Wu RX, Yu Y, Gao LN, Chen FM. Cell responses to conditioned media produced by patient-matched stem cells derived from healthy and inflamed periodontal ligament tissues. J Periodontol. 2016;87(5): e53-63.

44. Liu D, Yi C, Zhang D, Zhang J, Yang M. Inhibition of proliferation and differentiation of mesenchymal stem cells by carboxylated carbon nanotubes. ACS Nano. 2010;4(4):2185-95.

45. Liu TM, Lee EH. Transcriptional regulatory cascades in Runx2-dependent bone development. Tissue Eng Part B Rev. 2013;19(3):254-63.

46. Duan P, Bonewald LF. The role of the wnt/beta-catenin signaling pathway in formation and maintenance of bone and teeth. Int J Biochem Cell Biol. 2016;77(Pt A):23-9.

47. Gaur T, Lengner CJ, Hovhannisyan H, Bhat RA, Bodine PV, Komm BS, Javed A, van Wijnen AJ, Stein JL, Stein GS, et al. Canonical WNT signaling promotes osteogenesis by directly stimulating Runx2 gene expression. J Biol Chem. 2005;280(39):33132-40.

48. Liang L, Zhou W, Yang N, Yu J, Liu H. ET-1 promotes differentiation of periodontal ligament stem cells into osteoblasts through ETR, MAPK, and Wnt/beta-catenin signaling pathways under inflammatory microenvironment. Mediat Inflamm. 2016;2016:8467849.

49. Liu H, Zheng J, Zheng T, Wang P. Exendin-4 regulates Wnt and NF-kappaB signaling in lipopolysaccharide-induced human periodontal ligament stem cells to promote osteogenic differentiation. Int Immunopharmacol. 2019;75:105801.

50. Cao Z, Li C, Zhu G. Inhibitory effects of baicalin on IL-1 beta- induced MMP1/TIMP-1 and its stimulated effect on collagen-I production in human periodontal ligament cells. Eur J Pharmacol. 2010;641(1):1-6.

51. Wang GF, Wu ZF, Wan L, Wang QT, Chen FM. Influence of baicalin on the expression of receptor activator of nuclear factor-kappaB ligand in cultured human periodontal ligament cells. Pharmacology. 2006;77(2):71-7. 
52. Darveau RP, Pham TT, Lemley K, Reife RA, Bainbridge BW, Coats SR, Howald WN, Way SS, Hajjar AM. Porphyromonas gingivalis lipopolysaccharide contains multiple lipid a species that functionally interact with both toll-like receptors 2 and 4 . Infect Immun.

2004;72(9):5041-51.

53. Yamaji Y, Kubota T, Sasaguri K, Sato S, Suzuki Y, Kumada H, Umemoto T. Inflammatory cytokine gene expression in human periodontal ligament fibroblasts stimulated with bacterial lipopolysaccharides. Infect Immun. 1995;63(9):3576-81.

54. Kim JE, Takanche JS, Yun BS, Yi HK. Anti-inflammatory character of Phelligridin D modulates periodontal regeneration in lipopolysaccharideinduced human periodontal ligament cells. J Periodontal Res. 2018;53(5): 816-24.

55. Nebel D, Svensson D, Arosenius K, Larsson E, Jonsson D, Nilsson BO. 1alpha,25-dihydroxyvitamin D3 promotes osteogenic activity and downregulates proinflammatory cytokine expression in human periodontal ligament cells. J Periodontal Res. 2015;50(5):666-73.

56. Xing Y, Zhang Y, Jia L, Xu X. Lipopolysaccharide from Escherichia coli stimulates osteogenic differentiation of human periodontal ligament stem cells through Wnt/ß-catenin-induced TAZ elevation. Mol Oral Microbiol. 2019;34(1):1-13.

\section{Publisher's Note}

Springer Nature remains neutral with regard to jurisdictional claims in published maps and institutional affiliations.

Ready to submit your research? Choose BMC and benefit from:

- fast, convenient online submission

- thorough peer review by experienced researchers in your field

- rapid publication on acceptance

- support for research data, including large and complex data types

- gold Open Access which fosters wider collaboration and increased citations

- maximum visibility for your research: over $100 \mathrm{M}$ website views per year

At BMC, research is always in progress.

Learn more biomedcentral.com/submissions 